\title{
Glypican-1 Nanoliposomes for Potentiating Growth Factor Activity in Therapeutic Angiogenesis
}

\author{
Anthony J. Monteforte ${ }^{1}$, Brian Lam ${ }^{1}$, Subhamoy Das ${ }^{1}$, Somshuvra Mukhopadhyay ${ }^{2,3,4}$, \\ Catherine S. Wright ${ }^{4}$, Patricia E. Martin ${ }^{4}$, Andrew K. Dunn ${ }^{1}$ and Aaron B. Baker ${ }^{1,4,6}$
}

${ }^{1}$ Department of Biomedical Engineering, University of Texas at Austin, Austin, TX

${ }^{2}$ Division of Pharmacology \& Toxicology, University of Texas at Austin, Austin, TX

${ }^{3}$ Institute for Neuroscience, University of Texas at Austin, Austin, TX

${ }^{4}$ Institute for Cellular and Molecular Biology, University of Texas at Austin, Austin, TX

${ }^{5}$ Diabetes Research Group, Department of Life Sciences and Institute for Applied Health

Research, Glasgow Caledonian University, Glasgow G4 0BA, UK

${ }^{6}$ The Institute for Computational Engineering and Sciences, University of Texas at Austin, Austin, TX

Total Figures: 8 + 10 Supplemental Figures

Total Tables: 1

Running Title: Glypisomes for Therapeutic Angiogenesis

Correspondence to:

Aaron B. Baker

University of Texas at Austin

Department of Biomedical Engineering

1 University Station

BME 5.202D, C0800

Austin, TX 78712

Phone: 512-232-7114

E-mail: abbaker@austin.utexas.edu 


\section{ABSTRACT}

Therapeutic angiogenesis is a highly appealing concept for treating tissues that become ischemic due to vascular disease. A major barrier to the clinical translation of angiogenic therapies is that the patients that are in the greatest need of these treatments often have long term disease states and co-morbidities, such as diabetes and obesity, that make them resistant to angiogenic stimuli. In this study, we identified that human patients with type 2 diabetes have reduced levels of glypican-1 in the blood vessels of their skin. The lack of this key co-receptor in the tissue may make the application of exogenous angiogenic growth factors or cell therapies ineffective. We created a novel therapeutic enhancer for growth factor activity consisting of glypican-1 delivered in a nanoliposomal carrier (a "glypisome”). Here, we demonstrate that glypisomes enhance FGF2 mediated endothelial cell proliferation, migration and tube formation. In addition, glypisomes enhance FGF-2 trafficking by increasing both uptake and endosomal processing. We encapsulated FGF-2 or FGF-2 with glypisomes in alginate beads and used these to deliver localized growth factor therapy in a murine hind limb ischemia model. Co-delivery of glypisomes with FGF-2 markedly increased the recovery of perfusion and vessel formation in ischemic hind limbs of wild type and diabetic mice in comparison to mice treated with FGF-2 alone. Together, our findings support that glypisomes are effective means for enhancing growth factor activity and may improve the response to local angiogenic growth factor therapies for ischemia.

KEYWORDS: angiogenesis, neovascularization, ischemia, fibroblast growth factor-2 (FGF-2), vascular endothelial growth factor (VEGF), glypican-1, proteoliposomes, peripheral arterial disease. 


\section{INTRODUCTION}

Peripheral arterial disease (PAD) affects over 200 million people worldwide and is estimated to afflict approximately $16 \%$ of the general population over 65 years of age. Severe PAD has serious consequences for patients including the formation of non-healing ulcers, pain from intermittent claudication and, ultimately, increased risk for limb amputation. The current clinical treatments for PAD include surgical revascularization with bypass grafting or endartectomy, or percutaneous interventions such as angioplasty, stenting and atherectomy. However, these procedures cannot be performed in a significant portion of patients and many do not respond to these therapies [1]. An alternative approach for treating ischemic disease is to stimulate the body to create new vasculature to restore blood flow through its own regenerative processes. Several approaches have been explored to this end including the delivery of progenitor cells [2], viral vectors to express growth factor/angiogenic transcription factor genes [2-11] or through the delivery of growth factors $[3,12,13]$. Growth factors as protein therapeutics for ischemia have potential advantages from regulatory, production and delivery perspectives. However, in practice angiogenic growth factor therapies, both through delivered proteins and genes, have led to disappointing results in clinical trials [14]. Thus, while the concept of therapeutic angiogenesis has great promise, there are no current treatments that are capable of stimulating neovascularization in the context of human ischemic disease.

The presence of diabetes increases the risk of developing PAD by two-fold and increases the rate of progression of the disease [1]. Diabetic patients are 5-10 fold more likely to need limb amputation due to PAD [15]. The disease processes that drive development of ischemia may also induce disruptions in the pathways that are critical to mounting an effective angiogenic response to growth factor therapy. Insulin resistance is a hallmark of diabetic disease and, similarly, longterm ischemic vascular disease in aged humans may also represent a state in which the body can no longer respond effectively to growth factors such as FGF-2 and VEGF in ischemic tissues. We have recently shown that many of the heparan sulfate proteoglycans (HSPGs) that serve as co-receptors for the FGF-2 and VEGF families of growth factors are expressed at lower levels in ob/ob mice than wild type (WT) mice [16]. In addition, heparanase expression is increased in cells treated with fatty acids and in animals on a high fat diet [17-20], in atherosclerotic plaques [20-22] and following stenting or vascular injury [23, 24]. Heparanase is an enzyme that can degrade the heparan sulfate chains on cell surface proteoglycans $[25,26]$, ultimately leading to 
enhanced surface shedding of these molecules and loss of their activity as co-receptors for growth factor signaling [27, 28]. Thus, many disease states and pathophysiological processes lead to the loss of HSPGs from the cell surface.

In this study, we have examined the expression of the cell surface proteoglycan glypican-1 in disease and explored its use as a therapeutic enhancer for angiogenic growth factor delivery. The glypicans are distinguished from other cell surface HSPGs by their linkage to the membrane through a glycosylphosphatidylinositol (GPI) anchor. This GPI linker enables the phospholipase mediated-shedding of glypicans and drives preferential localization of the protein in cholesterolrich lipid rafts [29]. These properties allow glypicans to associate with calveolae [30], control endocytosis/recycling and transcellular transport [31-34], regulate the formation of morphogen gradients [35, 36] and facilitate growth factor cell signaling [37-40]. Glypican-1, in particular, is highly expressed in gliomas and their associated vasculature [41, 42]. A hallmark of gliomas is vigorous angiogenic response to the tumor that drives neovascularization through multiple mechanisms [31-34]. Glypican-1 has a key role in the growth, metastasis and angiogenic properties of gliomas [41-44]. In addition, glypican-1 is the most prevalent member of the glypican family expressed in endothelial cells and the vascular system [45]. It can act as a coreceptor/modulator for many angiogenic factors including members of the FGF and VEGF growth factor families [41, 45-48]. Several studies also have shown that glypican-1 can stimulate cell cycle progression in endothelial cells [44, 49]. In this work, we show that glypican-1 is lost in the blood vessels of skin samples from human patients with type 2 diabetes. We developed a novel therapeutic that consists of glypican-1 embedded in a nanoliposomal carrier (a "glypisome") that can be locally delivered to ischemic tissues from an alginate gel to potentiate the angiogenic response to localized growth factor therapies in diseased tissues. We demonstrate that this therapeutic enhancer improves the in vitro activity of FGF-2 and VEGF in endothelial cells through multiple mechanisms. When locally delivered from an alginate gel, glypisomes also enhance the therapeutic potential of FGF-2 therapy leading to increased revascularization of ischemic limbs in wild type and diabetic mice. 


\section{MATERIALS AND METHODS}

2.1. Cell culture. Human umbilical vein endothelial cells (HUVECs) were cultured in MCDB131 media with 7.5\% FBS, EGM-2 supplements (Lonza), L-glutamine and antibiotics. Endothelial cells that were used for the experiments did not exceed passage six. HeLa cells (ATCC) were cultured in high glucose DMEM with 10\% FBS, L-glutamine and antibiotics. For lentiviral production, HEK293T cells were cultured in DMEM with sodium pyruvate, 10\% FBS, L-glutamine and antibiotics.

2.2. FGF-2 trafficking and Rab co-localization assays. Constructs for expressing GFP-Rab5, GFP-Rab7 and GFP-Rab11 were as described previously [50, 51]. A construct to express GFPRab11 was created using similar methods. For studying the effect of glypisomes on endosomal trafficking, HEK 293 cells were plated on glass bottom dishes and transfected with the contructs using lipofectamine 2000 (Life Technologies, inc.). We conjugated Alexa Fluor 594 to FGF-2 while it was bound to heparin column to prevent loss of activity as described previously [52]. Twenty-four hours after transfection, $27 \mathrm{ng} / \mathrm{ml}$ of labeled FGF-2 was added. The cells were fixed at various time points after the addition of FGF-2 and then imaged using a laser scanning confocal microscope (LSM 710; Zeiss). The percentage Rab-positive endosomes that also contained FGF-2 was quantified using 10 cells at each time point (all Rab-positive endosomes were counted for each cell). The total fluorescence intensity and nuclear intensity of FGF-2 was calculated by selecting the whole cell or DAPI-stained nucleus and measuring intensity in exposure normalized images using Photoshop software (Adobe).

2.3. Cell proliferation assay. Endothelial cells were passaged into a 96-well plate and cultured in low serum media with 2\% FBS for 24 hours. Glypisomes and/or growth factors were then added to cells. After 24 hours, BrdU was added to the cells and proliferation was assessed 12 hours later using a colorimetric BrdU assay (Cell Signaling, Inc.). For the temporal bead release study 27,000 endothelial cells/well were plated in a 12 well plate and an alginate bead containing either FGF-2 or glypisomes was added to the well, at each time point the number of cells were counted using a hemocytometer and normalized to the initial cell count. 
2.4. Wound healing cell migration assay. Endothelial cells were grown to confluence and then cultured in low serum media (2\% FBS) for 24 hours. A cell scraper was used to create a wound in each well. The glypisomes were added with FGF-2 (10 ng/ml) or VEGF-A (10 ng/ml) immediately after wounding of the monolayer. The wounds were imaged along their entire length using an inverted, phase-contrast microscope with digital camera immediately after wounding and six hours later. The average gap was calculated using Metamorph software (Molecular Devices) the rate of wound closure was calculated by taking the difference between the two measurements.

2.5. In vitro angiogenic differentiation assay. Multi-well culture plates were coated with growth factor depleted Matrigel (BD Biosciences) and allowed to gel for one hour at $37^{\circ} \mathrm{C}$. In each well, endothelial cells were plated at a concentration of $2 \times 10^{4}$ cells per well in 24-well plates. The cells were treated with the various combinations of glypisomes and growth factors. The cells were imaged after 16 hours and then quantified for average tubule length, number of tubules, and number of tubule branching points were then quantified using Metamorph software (Molecular Devices).

2.6. Preparation of glypisomes. For the production of recombinant glypican-1, HeLa cells were transduced with a lentiviral vector with constitutive expression of 6x-His tagged glypican-1. Viruses were produced in HEK 293T cells using a human lentiviral packageing system according to the manufacturer's instructions (Genecopoeia). The cells were then selected with puromycin to create a stable cell line. The cells were lysed in a buffer containing $20 \mathrm{mM}$ Tris (pH 8.0), 150 $\mathrm{mM} \mathrm{NaCl}, 1 \%$ Triton X-100, $2 \mathrm{mM}$ sodium orthovanadate, $2 \mathrm{mM}$ PMSF, $50 \mathrm{mM} \mathrm{NaF}$, and protease inhibitors (Roche) at room temperature. Glypican-1 was then isolated using a $\mathrm{Ni}$ Sepharose column (His SpinTrap Kit; GE Healthcare). The buffer was exchanged and protein concentrated using Centriprep concentrators (Millipore). The final working concentration of protein was found to be $61 \mu \mathrm{g} / \mathrm{ml}$ using a micro BCA Protein Assay kit (Thermo Scientific). Purity of the protein was confirmed by SDS-PAGE followed silver staining of the gel (Supplemental Figure 1). We then mixed lipid stock solutions (10 mg/ml in chloroform) of 1,2dioleoyl-sn-glycero-3-phosphocholine (DOPC), 1,2-dioleoyl-sn-glycero-3-phosphoethanolamine (DOPE), cholesterol and sphingomyelin in a volumetric ratio of 2:1:1:1, respectively, in a round 
bottom flask. The solvent was removed using rotary evaporation and the lipid film further dried under argon gas. The film was resuspended in HEPES buffer by vortexing, sonication and three cycles of freeze thawing (total lipid concentration of $12.3 \mathrm{mM}$ ). The lipid solution was then extruded through a $400 \mathrm{~nm}$ polycarbonate filter membrane to create liposomes. The liposome solution was mixed to varying volumes of glypican-1 (61 $\mu \mathrm{g} / \mathrm{ml})$ and $1 \% \mathrm{n}$-octyl- $\beta-\mathrm{D}-$ glucopyranoside. The detergent was extracted to allow glypican-1 incorporation into the liposome membrane through progressive dilution, dialysis and zeolite treatment. Every 30 minutes the concentration of the solution was reduced $10 \%$ by adding PBS to a final concentration of $40 \%$ of the original solution. The excess protein and detergent were then removed extensive dialysis and the final solution treated with Biobeads (SM-2; Bio-Rad) to remove any residual detergent. The final concentration of the glypisomes contained an 83.3 $\mu \mathrm{g} / \mathrm{ml}$ of the lipid solution and $4.88 \mu \mathrm{g} / \mathrm{ml}$ of the glypican- 1 .

2.7. Immunoblotting and silver staining. Following the various treatments, cells were lysed in a solution containing the following: $20 \mathrm{mM}$ Tris at $\mathrm{pH} 8,150 \mathrm{mM} \mathrm{NaCl}, 1 \%$ triton, $0.1 \%$ SDS, 2 $\mathrm{mM}$ sodium orthovanadate, $2 \mathrm{mM}$ PMSF, $50 \mathrm{mM} \mathrm{NaF}$, and protease inhibitors (Roche). For tissue samples, cryosections were collected, and the sections placed into the above lysis buffer and homogenized using a Tissuelyzer device (Qiagen). Lysates from cells or tissues were clarified with centrifugation, normalized for protein concentration and separated on Nupage Novex 10\% Bis-Tris gels (Lifetech). Samples were then transferred using the iBlot System to a nitrocellulose membrane using the iBlot Gel Transfer Stacks (Life Technologies). The membrane was washed twice in phosphate buffered saline with $0.1 \%$ tween (PBST). The membranes were then incubated at $4^{\circ} \mathrm{C}$ overnight in a 1:500 dilution of glypican-1 antibody (Abcam), phosphoERK, ERK, phosphor-AKT or AKT antibodies (Cell Signaling). Membranes were washed twice in PBST and incubated with an appropriate HRP-linked secondary antibody at 1:5000 dilution for two hours at room temperature. The membranes were washed extensively in PBST and imaged using the Femto Maximum Sensitivity Substrate (Thermo Scientific) with a FluorChem HD2 System (Cell Biosciences). Silver staining was performed on gels using a silver staining kit according to the manufacturer's instructions (Thermo Scientific). 
2.8. Liposome characterization. The size and dispersion of the isolated glypican- 1 and liposomes was characterized by dynamic light scattering (Malvern Zetasizer Nano ZS). Calibration was performed using $54 \mathrm{~nm}$ polystyrene particles. Results shown are the average of 50 measurements. To visualize the glypisomes, carbon support grids (300 mesh Cu; EM Sciences) were treated with glow discharge at $50 \mathrm{~mA}$ for two minutes (Emitech K100x; Quorum Technologies). The samples were then applied to the grids and the excess liquid removed with a filter paper. The grids were then stained with a $2 \%$ uranyl acetate solution and imaged using an FEI Tecnai Transmission Electron Microscope (TEM).

\subsection{Preparation of alginate beads, measurement of release kinetics and scanning electron} microscopy. Equal volumes of a $4 \%$ sodium alginate solution and a $0.85 \% \mathrm{NaCl}$ solution were mixed together. The glypisomes (1:100 dilution) and FGF $(1.5 \mu \mathrm{g} / 100 \mu \mathrm{l})$ were added to this solution. Beads were created by controlled extrusion into a $1.1 \% \mathrm{CaCl}_{2}$ solution through a $30 \mathrm{G}$ needle using a syringe pump. The beads were allowed to crosslink for 1 hour at $4^{\circ} \mathrm{C}$. For measuring the release kinetics, alginate beads were put into plastic scintillation vials containing PBS with calcium and magnesium chloride. At various time points the liquid was completely removed, replaced and the harvested liquid frozen at -80C. The FGF-2 released was quantified using ELISA (R\&D Systems). Glypican-1 release was quantified using an ELISA (Ray Biotech). To examine the morphology of the alginate gels, the samples were flash frozen in liquid nitrogen $\left(-195^{\circ} \mathrm{C}\right)$ and lyophilized overnight $\left(-110^{\circ} \mathrm{C} ; 0.0005 \mathrm{mbar}\right)$ in scintillation vials. The freeze-dried gels were sputter coated with gold discharge for 30 seconds and then loaded on the stage to image them under the scanning electron microscope (FEI Quanta 650 ESEM) with an acceleration voltage of $10 \mathrm{kV}$.

2.10. Animal studies. Studies involving animals were performed with the approval of the University of Texas at Austin Institutional Animal Care and Use Committee (IACUC) and in accordance with NIH guidelines for animal care. Wild-type mice or ob/ob mice (B6.Cg-Lep ${ }^{\mathrm{ob}} / \mathrm{J}$; Jackson Labs) were used in the studies. The ob/ob mice were fed a high fat diet (Research Diets D12331) for 10 weeks prior to performing experiments. To perform the hind limb ischemia studies, mice were anesthetized with $2 \%$ isofluorane gas and a longitudinal incision was made in the inguinal region of the right thigh. The femoral artery was separated from the femoral vein 
and nerve, and then double ligated with 6-0 silk sutures at two locations and the artery severed at each ligation. Alginate beads containing the various treatments were then implanted in the pocket created by the surgery. A total of $3 \mu \mathrm{g}$ FGF-2 and $19.5 \mathrm{ng}$ of glypican-1 was implanted per mouse. The incision was then closed with vicryl sutures. Relative blood flow between the ischemic and contralateral control limb was measured at days 1, 3, 5, 7, and 14 using laser speckle imaging as described previously [53]. At day 14, the mice were sacrificed and the thigh/calf muscles were harvested and frozen in liquid $\mathrm{N}_{2}$ cooled isopentane.

2.11. Human tissues, immunostaining and histological analysis. Human skin samples were obtained from the Glasgow Caledonian University Skin Research Tissue Bank, Glasgow UK. The tissue bank has NHS research ethics to supply human skin for research (REC REF: 11/S1402/2). Samples were formalin fixed and embedded in paraffin following standard procedures prior to sectioning. Six skin samples were collected from non-diabetic patients and seven skin samples from those with type 2 diabetes. In the hind limb ischemia mouse model, the thigh and calf muscles were harvested from the mice, fixed in formalin and then processed for paraffin sectioning. Both human skin and mouse tissue samples were cut with a microtome to obtain $6 \mu \mathrm{m}$ thick sections were then stained using hematoxylin and eosin for overall morphology. The sections were deparaffinized and treated for 3 hours with antigen retrieval solution (Dako) at $80^{\circ} \mathrm{C}$. The sections were cooled to room temperature and blocked with $20 \%$ fetal bovine serum for 45 minutes and then immunostained with the Envision+ Dual Link Kit (Dako) using a 1:100 dilution of primary antibody to PECAM-1 (Millipore) or a 1:500 dilution of primary antibody to glypican-1 (Thermo Scientific). Following staining, the samples were imaged using a bright field microscope (Meiji). For fluorescent immunostaining, the sections were treated as described as above but then treated with primary antibodies to glypican-1, PECAM-1 or a-SMA (Abcam). The staining was detected after treatment with fluorescently labeled secondary antibodies. For murine samples, the number of fibers with ischemic changes was quantified in a minimum of three low magnification images per mouse. Histological sections from the contralateral limb were used as a control for artifacts of histological processing. Following immunostaining for PECAM-1, the number of small and large vessels was counted in images from each of the mice. For human skin samples, we defined DAB intensity for positive 
staining and counted the number of cells positive for glypican-1 staining in the arterioles in the skin.

2.12. Statistical Analysis. All results are shown as mean \pm standard error of the mean. Comparisons between only two groups were performed using a two-tailed Student's t-test. Multiple comparisons between groups were analyzed using a two-way ANOVA followed by Dunnett post-hoc testing. A two-tailed probability value of $\mathrm{p}<0.05$ was considered statistically significant. 


\section{RESULTS}

3.1. Glypican-1 is reduced in the blood vessels of skin tissues from diabetic patients. Our recent studies have shown that there are reduced protein levels of the syndecan family of cell surface heparan sulfate proteoglycans in diabetic mice on a high fat diet [16]. This finding led us to hypothesize that loss of growth factor co-receptors, including glypican-1, is a key mechanism in diabetes-mediated growth factor resistance. To test if a similar phenomenon was happening in humans with diabetes, we collected skin samples from human patients and immunostained sections from these samples for glypican-1 (Fig. 1A; Table 1). This analysis revealed a significant reduction in the number of glypican-1 positive cells in the arterioles from the skin of patients with type 2 diabetes (Fig. 1B). Consistent with these findings, we performed western blotting on tissues from ob/ob and wild type mice that had been exposed to a high fat diet to 10 weeks and found a reduced amount of glypican- 1 in ob/ob mice versus wild type mice with a high fat diet (Supplemental Fig. 2). Glypican-1 serves as a co-receptor for angiogenic growth factors and, consequently, its loss may lead to reduced growth factor responsiveness. To overcome this mechanism of therapeutic resistance, we created a protein therapeutic formulation of glypican-1 by embedding it in the lipid membrane of a liposome that allows it to be codelivered with growth factors ("glypisomes"; Fig. 2).

\subsection{Synthesis of glypisomes and characterization of release kinetics from an alginate carrier.}

To create glypisomes, we produced and isolated recombinant glypican- 1 and then embedded the proteins in the membranes of $400-\mathrm{nm}$ liposomes using the progressive detergent extraction method (Fig. 3A; Supplemental Fig. 1). For delivery, the glypisomes were encapsulated in an alginate gel (Fig. 3B). We created a set of glypisomes with varying lipid:protein ratio, ranging from 100\% liposomes with no glypican-1 (L100:G0) to the isolated glypican-1 protein alone (L0:G100). We examined the size distribution of the glypisomes using transmission electron microscopy (TEM) and dynamic light scattering (DLS; Fig. 3C). As anticipated, the liposomes and glypisomes had a size distribution near to the 400-nm diameter of the liposomes following the initial extrusion step. Surprisingly, the isolated glypican-1 alone had a size of around $190 \mathrm{~nm}$ in diameter. This result suggests that self-association occurs for isolated glypican-1 and/or the co-isolation of lipids that creates an aggregate that has a diameter similar to that of the liposomal 
embedding particles. We analyzed the isolated glypican-1 for cholesterol to see if there was association of lipid rafts with the glypican-1 and this analysis revealed undetectable levels of cholesterol (data not shown). We encapsulated the mid-range composition glypisomes (L20:G80) in an alginate gel combined with FGF-2 and measured the release of FGF-2 over time using ELISA. The incorporation of glypisomes led to a similar in comparison to FGF-2 alone, with a slight increase in the release of FGF-2 for the later time points out to 14 days (Fig. 3D). The release profile for glypican-1 from these beads was slower than that of FGF-2, likely reflecting the larger size of the proteoliposome (Fig. 3E).

\subsection{Glypisomes enhance proliferation and migration of endothelial cells stimulated with}

FGF-2 or VEGF. To optimize the effects of glypisome composition on activity, we measured the ability of glypisomes to enhance FGF-2 or VEGF activity in a proliferation assay. For FGF2, glypisomes with higher levels of glypican-1 enhanced FGF-2 induced proliferation of endothelial cells by nearly three-fold (Fig. 4A). Notably, the liposome alone had an enhancing effect on proliferation in comparison to FGF-2, an effect we had observed in previous studies using a similar liposome formulation [52]. Interestingly, isolated glypican-1 also increased proliferation but not to the full extent as the mid-range composition glypisomes. In contrast, VEGF-induced proliferation was not significantly altered by the addition of liposome or glypisomes (Fig. 4A). The isolated glypican-1 reduced the proliferation in response to VEGF to baseline levels. Glypisomes and isolated glypican-1 had only small effects on endothelial proliferation in the absence of growth factors (Supplemental Fig. 3). Human endothelial cells treated with glypisomes or FGF-2 with glypisomes encapsulated within an alginate bead show a significant increase in proliferation after one day and FGF-2 and glypisomes show a significant increase in proliferation over alginate alone and glypisomes after the second day (Fig. 4B). In addition, we examined the effect of the glypisomes on the migration of endothelial cells in an in vitro scratch wound assay. We found that for the L20:G80 composition of glypisomes there was no enhancement of FGF-2 induced migration but VEGF induced migration was increased (Fig. 4C). We found that glypisomes in the absence of growth factors have no significant effect on endothelial migration (Supplemental Fig. 4). 
3.4. Glypisomes enhance FGF-2 induced in vitro tube formation. We next examined the effect of glypisome treatment on growth factor activity in an in vitro tube formation assay of angiogenic differentiation. Endothelial cells were grown on growth factor depleted matrigel, serum starved and then treated with growth factors or growth factors in combination with the glypisomes of varying composition. For glypisomes in combination with FGF-2 there was a marked increase in branch points, tube length and number of tubes formed in cells treated with the glypisomes with higher levels of glypican-1 (Fig. 5). When glypisomes were delivered with VEGF we found they enhanced tube length with midrange concentrations of glypican-1 (60:40 and 40:60 lipid to protein ratio; Supplemental Fig. 5). Isolated glypican-1 also increased VEGFinduced differentiation but to a lesser extent than the midrange composition glypisomes. There were no significant alterations in the number of branch points and number of tubes with glypisome treatment in combination with VEGF.

\subsection{Glypisomes increase FGF-2 induced signaling through the AKT pathway and enhance} both FGF-2 uptake and endosomal trafficking. We examined whether glypican-1 proteoliposomes could increase the downstream signaling pathways induced by FGF-2. For the ERK1/2 pathway, we found that the cell signaling response with and without glypisomes was very similar (Supplemental Fig. 6A). However, for the AKT pathway there was a moderate increase in the AKT signaling after 60 minutes (Supplemental Fig. 6A). Notably, neither pathway was appreciably activated by the glypisomes without the growth factor. To further explore the mechanism of glypisome enhancement of FGF-2 activity, we treated cells expressing GFP-labeled versions of the endosomal trafficking proteins Rab5, Rab7 or Rab11 with flurophore-labeled FGF-2 (Fig. 6). These proteins are markers of early (Rab5) and late (Rab7) endosomes as well as endosomal recycling (Rab11). We found increased numbers of Rab5 and Rab7 positive endosomes that contained FGF-2 at all time points after 15 or 30 minutes, respectively, with treatment with glypisomes. At 120 minutes, there were increased numbers of Rab11 positive endosomes that contained FGF-2 in the glypisome treated group. We also measured the total cell fluorescence intensity for FGF-2 and found this was moderately increased for the glypisome group (Supplemental Fig. 7A). Finally, we measured the intensity of FGF-2 in the nucleus for the cells and found increased nuclear localization at the 15-minute time point for the glypisome group (Supplemental Fig. 7B). Together, these results support the notion that 
glypisomes have activity through a moderate enhancing effect on AKT signaling, by increasing FGF-2 uptake and through enhancing endosomal processing of FGF-2.

\subsection{Local delivery of glypisomes enhances FGF-2 induced revascularization in the ischemic} hind limb model in wild type and diabetic mice. Both exogenous FGF-2 and VEGF have been shown to enhance revascularization in animal models of ischemia [54, 55]. Based on our in vitro studies, glypisomes appeared to be more effective in enhancing FGF-2 activity in comparison to VEGF. We induced hind limb ischemia in mice by ligating the femoral artery and implanted alginate beads that encapsulated FGF-2 or FGF-2 with the glypisomes (L20:P80; Fig. 7A). Perfusion in the ischemic limb and contralateral control limb was monitored using laser speckle imaging over 14 days. We found that glypisomes enhanced FGF-2 activity and lead to nearly twice the relative perfusion in the ischemic limb of glypisome treated mice after 14 days in comparison to those treated with FGF-2 alone (Fig. 7B and Fig. 7C). Following the induction of ischemia, the muscle fibers can undergo ischemic changes that include the loss of fibers within the muscle. We quantified the number of fibers with ischemic changes that appeared as holes on histological analysis (Fig. 7D and Fig. 7F). Care was taken to differentiate the ischemic changes from artifacts of histological processing by the sections to those from the non-ischemic control limb for each animal. This analysis demonstrated a marked reduction in the ischemic changes in the glypisome treated mice. Immunohistochemical staining for endothelial cells (PECAM-1) and subsequent analysis revealed increased capillary density in the thigh and calf muscles, consistent with the increased perfusion observed by laser speckle imaging (Fig. 7E and Fig. 7G). An analysis of larger vessels (diameter $>25 \mu \mathrm{m}$ ) revealed increased arteriogenesis in the thigh muscle of glypisome treated mice (Fig. 7H). We repeated a similar study in ob/ob mice that had been treated with a high fat diet for 10 weeks. These animals have severe diabetes and obesity, and our group has shown them to be resistant to FGF-2 stimulation [16]. In these animals, we

found that glypisomes also improved perfusion recovery after femoral ligation (Fig. 8A,B) as well as increasing the vascularization (Fig. 8C-E). Consistent with these findings, we found that there were less signs of ischemia in the toes of the animals treated with glypisomes (Supplemental Fig. 8). To examine if glypican-1 delivery led to changes in glypican -1 in the tissues of the mice treated with glypisomes, we immunostained sections from the ischemic limbs of the mice for PECAM-1 and glypican-1. There was qualitatively higher diffuse glypican-1 
staining within the muscles of the mice that had glypican-1 delivery (Supplemental Fig. 9). We further confirmed increased numbers of arterioles in the muscles of the mice by performing coimmunostaining for PECAM- 1 and $\alpha$-SMA (Supplemental Fig. 10).

\section{DISCUSSION}

It has been challenging to develop angiogenic therapies that are effective in the context of human patients with disease. Here, we have shown that glypican-1 is reduced in the skin from patients with type 2 diabetes using immunohistochemical staining. This type of analysis is semiquantitative but the large difference between the patient groups would support that there may be significant alterations in glypican-1 levels in diabetic patients. The implication of these findings is that loss of glypican-1 may contribute to reduced tissue sensitivity to growth factors and inhibit the response to angiogenic therapies. Consequently, in spite of high levels of growth factor delivered in a therapeutic context the tissue would likely not be able to mount an effective angiogenic response. Our approach to overcoming this potential mechanism of growth factor resistance was to create a therapy that can deliver the missing glypican- 1 to the tissues without the need for gene therapies or the addition of exogenous cells. We delivered the glypican-1 embedded in a liposome membrane to simulate the endogenous glypican-1 presentation and to facilitate uptake of the protein. The formulation is stable when encapsulated in alginate and can be delivered locally to ischemic areas of the body. We show here that this method is very effective in increasing FGF-2 activity both in vitro and when locally delivered to the ischemic limb in wild type or diabetic mice.

Our in vitro studies revealed that glypisomes enhance growth factor activity but there were significant differences in the enhancement response between FGF-2 and VEGF. The proliferative response to FGF-2 was increased more by glypisomes than those stimulated with VEGF. In contrast, the cells simulated with VEGF and glypisomes had enhanced migration where FGF-2 simulated cells did not. Also, in the in vitro tube formation assay there were marked differences between VEGF and FGF stimulated network formation in the presence of glypisomes. The primary effect for cells treated with FGF-2 was an increase in branch points with a moderate

increase in tube length. However, for cells treated with VEGF the effect was primarily an increase in the length of the tubes without little or negative effects on the branch points. Glypican-1 has been shown to increase the activity of both these growth factors [38, 41, 45] and 
the diversity of the response to glypisomes may reflect the differences in the vascular networks that are induced FGF-2 and VEGF. FGF-2 is known to induce a strong proliferative response whereas VEGF has been associated with a stronger effect on phenotypic differentiation [56]. Glypisomes appeared to act more robustly on the FGF-2 pathway and enhanced all of the parameters measured in the tube formation assay. In contrast, glypisomes only increased the tube length in the tube formation assay with VEGF treatment.

Our studies demonstrated markedly increased uptake and concentration of FGF-2 in early (Rab5+) and late (Rab7+) endosomes. Prior studies have shown that glypican-1 serves as a low affinity receptor for FGF-2 that stabilizes the FGF-2/FGF receptor complex.[41] In addition, heparan sulfate proteoglycans can serve as mediators of cellular uptake of FGF-2 [57]. Endogenous cell surface glypican-1 is known to be constitutively endocytosed in many cell types where its heparan sulfate chains are partially degraded and the protein is transported to the Golgi for glycosylation [58]. Upon activation, the FGF-2/FGFR1 complex is internalized through a clathrin-mediated mechanism and, subsequently, FGFR1 is found localized with first early endosomes and then late endosomes [59]. Our findings of increased FGF-2 signaling, uptake and endosomal processing are consistent with the hypothesis that exogenous glypican-1 acts similarly to endogenous glypican-1 in stabilizing the receptor-growth factor complex. However, we observed increased activity of the liposome-embedded glypican-1 in comparison to free glypican-1. The liposomal component may facilitate the uptake of a larger complex of FGF2/FGFR-1 bound to a glypisome associated with additional FGF-2 not bound to the receptor complex on the other parts of the liposome. This concept would explain how the glypisome could increase uptake significantly while having only moderate effects on the initial FGF-2 signaling.

In addition to its effects on angiogenesis, glypican-1 has been found to be important in the regulation of myogenic satellite cell proliferation and differentiation [37, 60, 61]. Critical limb ischemia is often found in severe cases of peripheral vascular disease and leads to muscle atrophy in addition to many other symptoms [62]. Our study found a marked decrease in ischemia-induced muscle fiber damage in glypisome treated mice. Thus, the strategy of exogenously delivering glypican-1 may act through both angiogenic and muscle preserving mechanisms to improve the response to FGF therapy in-vivo. Consequently, glypisomes may 
present a therapeutic for treating both the deleterious effects of peripheral ischemia and provide additional myoprotective benefits by providing increased levels of gypican-1 in the muscle.

\section{CONCLUSIONS}

In summary, our study supports that glypisomes can markedly improve the effectiveness of growth factor therapies in the context of ischemia in the diabetic disease state. This conceptual archetype of delivering lost co-receptors to replace molecular alterations due to disease may be relevant to many potential therapeutic applications in which an undesirable change in tissue biology makes current therapies untenable. From the poor or equivocal results of many clinical trials using growth factors it is clear that growth factor therapy alone is not sufficient to treat human disease in many cases. Therefore, therapeutic enhancers such as glypisomes are needed to truly address the complexities of growth factor responsiveness in the context of human disease.

Acknowledgements. The authors would like to acknowledge support through the American Heart Association (10SDG2630139), the Welch Foundation (F-1836) and the NIH Director's New Innovator Grant (1DP2 OD008716-01) to A.B.B. This work was also supported by the NIH (EB-011556, NS-078791, NS-082518), NSF (CBET-0644638), American Heart Association (14EIA18970041), and the Coulter Foundation grants to A.K.D as well as NIH R00ES020844 to S.M. In addition, this work was supported by the Glasgow Caledonian University Skin Research Tissue Bank, Glasgow, UK (PEM and CSW). CSW is currently funded by the Dr Hadwen Trust (DHT) and did not participate in experiments involving animals, animal tissue, cells or cell lines or human embryonic stem cells. The authors would like to thank Drs. D. Busch and J. C. Stachowiak at the University of Texas at Austin for their aid in completing the TEM studies. 


\section{Figure Legends}

Figure 1. Glypican-1 is reduced in mice and human with diabetes. (A) Immunostaining for glypican-1 in skin samples from diabetic and non-diabetic patients. (B) Analysis of the number of DAB positive nuclei within arterioles within the skin. ${ }^{*}$ Statistically significant different from the no growth factor and growth factor alone groups (p < 0.05). Bar $=50 \square \mathrm{m}$.

Figure 2. Overall diagram of glypisome-based therapeutics for the treatment of ischemia. Recombinant glypican-1 is embedded in a liposome membrane using detergent extraction. This construct is then combined with growth factors and polymerized into an alginate gel for delivery.

Figure 3. Characterization of glypisomes and alginate delivery gel. (A) Visualization of glypisomes and isolated glypican-1 (GPC1) by transmission electron microscopy. Bar $=200$ $\square \mathrm{m}$. (B) Scanning electron microscopy visualization of the alginate gel after polymerization. Left bar $=100 \mu \mathrm{m}$ and right bar $=30 \mu \mathrm{m}$. (C) Dynamic light scattering of isolated glypican-1 (GPC1), liposomes and glypisomes. Glypisomes are listed as composition ratio between lipid (L\%) and protein (P\%). (D) Release kinetics of FGF-2 from alginate gel with and without glypisomes added. (E) Release kinetics of glypican-1 from alginate gels containing FGF-2 and glypisiomes.

\section{Figure 4. Glypisomes increase the proliferation and migration of endothelial cells.} Proliferation of human endothelial cells after stimulation with growth factors and glypisomes with varying ratios between lipid and recombinant glypcian-1 (GPC-1). (A) Endothelial cells were treated with $10 \mathrm{ng} / \mathrm{ml} \mathrm{FGF-2}$ or $10 \mathrm{ng} / \mathrm{ml} \mathrm{VEGF}_{165}$ and glypisomes, and then proliferation was measured from Brdu incorposation assay. ${ }^{*}$ Statistically significant difference between group and the no growth factor group ( $\mathrm{p}<0.05)$. ${ }^{\dagger}$ Statistically significant different from the no growth factor and growth factor alone groups (p < 0.05). (B) Human endothelial cells were treated with an alginate bead containing FGF-2 and/or glypisomes. The number of cells/well was counted at each time point. *Statistically significant difference between group and alginate alone $(\mathrm{p}<0.05)$. ${ }^{\dagger}$ Statistically significant difference between group and FGF-2 (p < 0.05). ${ }^{\ddagger}$ Statistically significant difference between group and glypisomes $(\mathrm{p}<0.05)$. (C) Wound closure after scratch wounding 
of an endothelial monolayer. Cells were treated with $10 \mathrm{ng} / \mathrm{ml} \mathrm{FGF-2} \mathrm{or} 10 \mathrm{ng} / \mathrm{ml}$ VEGF at the time of injury. The glypisomes (G1PL) with a lipid to glypican-1 protein ratio of 20:80 were used. * Statistically significant different from the no growth factor and growth factor alone groups (p<0.05). Bar $=200 \mu \mathrm{m}$.

Figure 5. Glypisomes enhance FGF-2 induced endothelial tube formation. Endothelial cells grown on growth factor reduced matrigel and the cells were treated with glypisomes with varying composition and $10 \mathrm{ng} / \mathrm{ml} \mathrm{FGF-2.} \mathrm{After} 16$ hours the formation of tubes was assessed by phase contrast microscopy. * Statistically significant difference between group and the no growth factor group $(\mathrm{p}<0.05){ }^{\dagger}{ }^{\dagger}$ Statistically significant difference from the no growth factor and growth factor alone groups $(\mathrm{p}<0.05)$. Bar $=200 \mu \mathrm{m}$.

Figure 6. Glypisomes increase endosomal trafficking of FGF-2 in HEK-293 cells. (A) Quantification of the percentage of Rab5, Rab7 or Rab11 positive (GFP) endosomes that contained FGF-2 after treatment of HEK-293 cells with various conditions. (B) Representative confocal images of cells treated with FGF-2 or FGF-2 with glypisomes at 120 min post treatment. *Statistically significant difference between the group and the FGF-2 only group at the same time point $(\mathrm{p}<0.05)$. Bar $=20 \mu \mathrm{m}$.

Figure 7. Glypisomes enhance therapeutic angiogenesis with FGF-2 in hind limb ischemia. (A) Optimal composition glypisomes were encapsulated in alginate beads. (B) Laser speckle contrast imaging was used to assess blood perfusion in the feet of the mice over time. Mice were given either alginate beads with FGF-2 or FGF-2 with glypisomes (G1PL). (C) Quantitative analysis of the perfusion of the feet after induction of hind limb ischemia. Relative blood flow was defined as the speckle contrast ratio between the ischemic limb and the control limb. *Statistically significant difference between the G1PL and G1PL + FGF-2 groups versus FGF-2 alone group at the same time point $(\mathrm{p}<0.05)$. (D) Histological analysis of the calf and thigh muscles of the ischemic limb after 14 days of treatment with FGF-2 or FGF-2 with glypisomes (G1PLs). (E) Immunohistochemical staining for blood vessels (PECAM-1) in the thigh and calf muscles. (F) Ischemic changes in the muscle fibers was reduced in the calf muscle with FGF-2 and glypisome treatment in comparison to FGF-2 alone. Ischemic changes included the loss of 
muscle fibers/altered morphology with the tissue. (G) Quantification of small vessel density. (H) Quantification of large vessel density (diameter $>25 \mathrm{~m}$ ). $\quad$ *Statistically significant difference with FGF-2 alone group $(\mathrm{p}<0.05)$. Bar $=100 \mu \mathrm{m}$.

\section{Figure 8. Glypisomes enhance therapeutic angiogenesis when delivered with FGF-2 in an} ob/ob hindlimb ischemia model. (A) Quantitative analysis of laser speckle contrast imaging gives relative blood flow analysis of perfusion measured over time. Relative flow defined as perfusion in the ischemic limb over the perfusion in the contralateral limb. (B) Representative laser speckle contrast images of each treatment group at 1, 3, 5, 7, and 14 days post injury. Right paw is from ischemic limb and left is the contralateral control. "Statistically significant difference with FGF-2 alone group ( $\mathrm{p}$ 0.05). (C) Representative H\&E stained muscle fiber cross-sections of the calf and thigh of the ischemic limb. (D) Immunohistochemical staining for PECAM in calf and thigh muscle tissue of the ischemic limb. (E) Quantification of small vessel density in the calf and thigh muscle tissue, and large vessel density in the thigh muscle tissue. ${ }^{*}$ Statistically significant difference from all other groups $(\mathrm{p}<0.05) .{ }^{* *}$ Statistically significant difference from alginate group $(\mathrm{p}<0.05)$. Bar $=100 \mu \mathrm{m}$. 
Figure 1

A Non-Diabetic
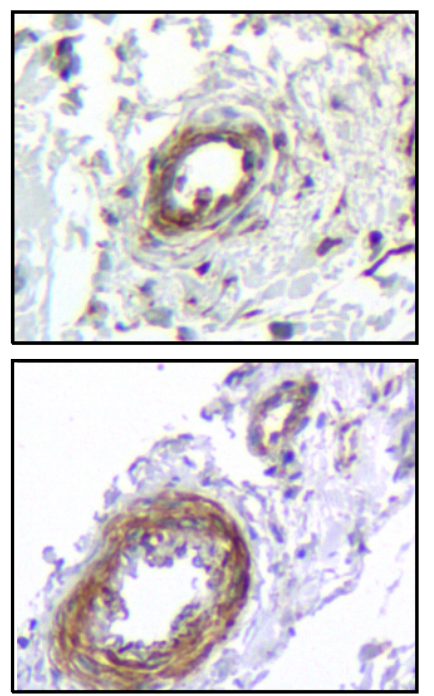

B

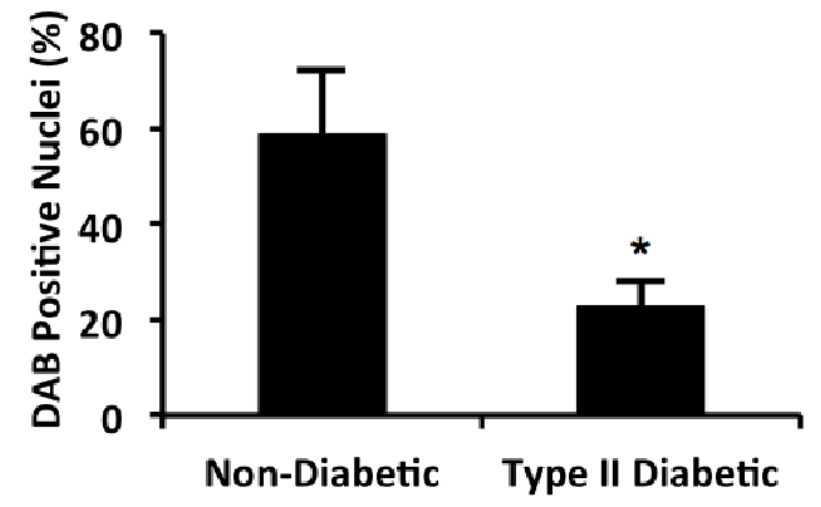

Diabetic
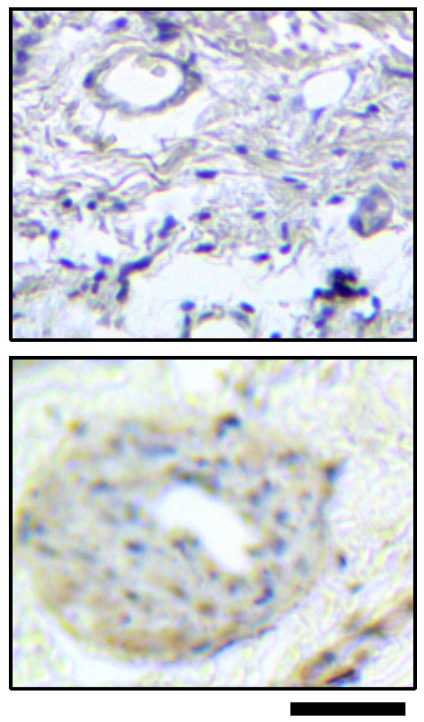
Figure 2

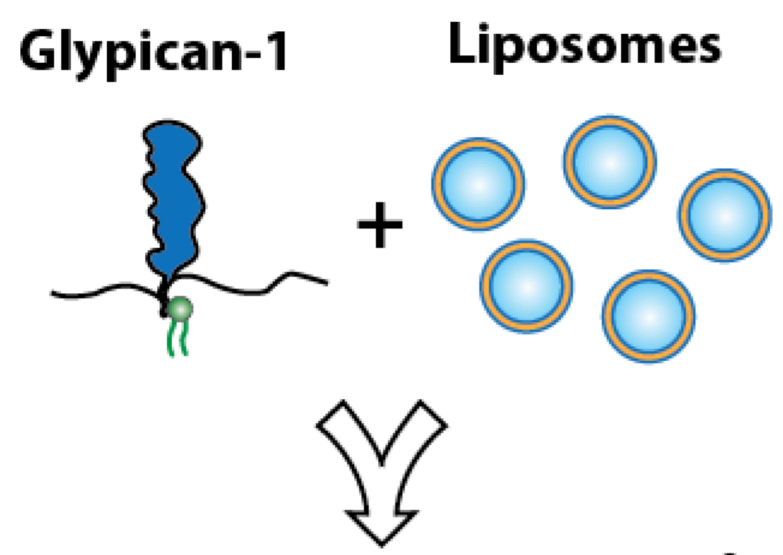

Glypisomes
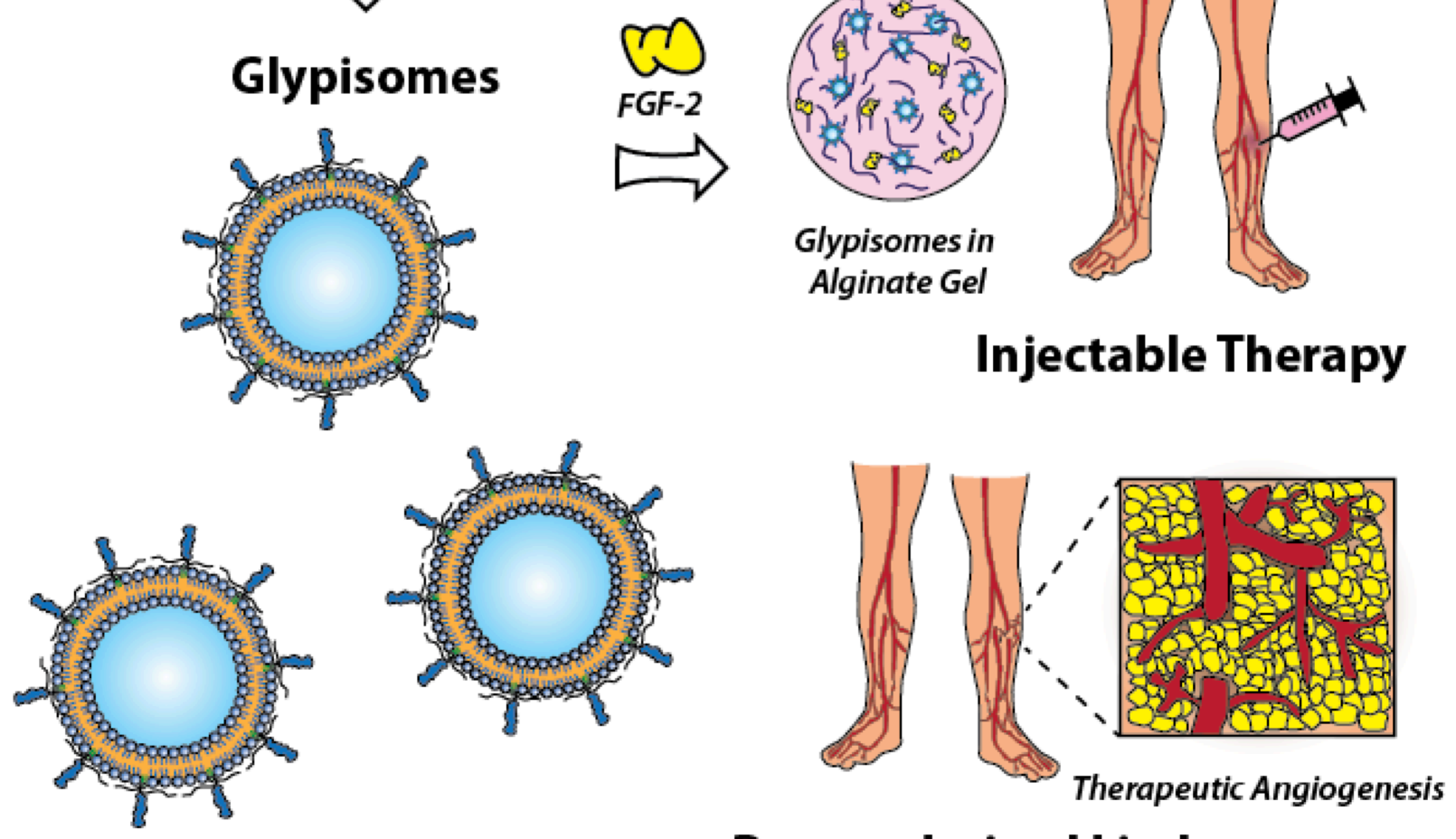

Revascularized Limb 
Figure 3
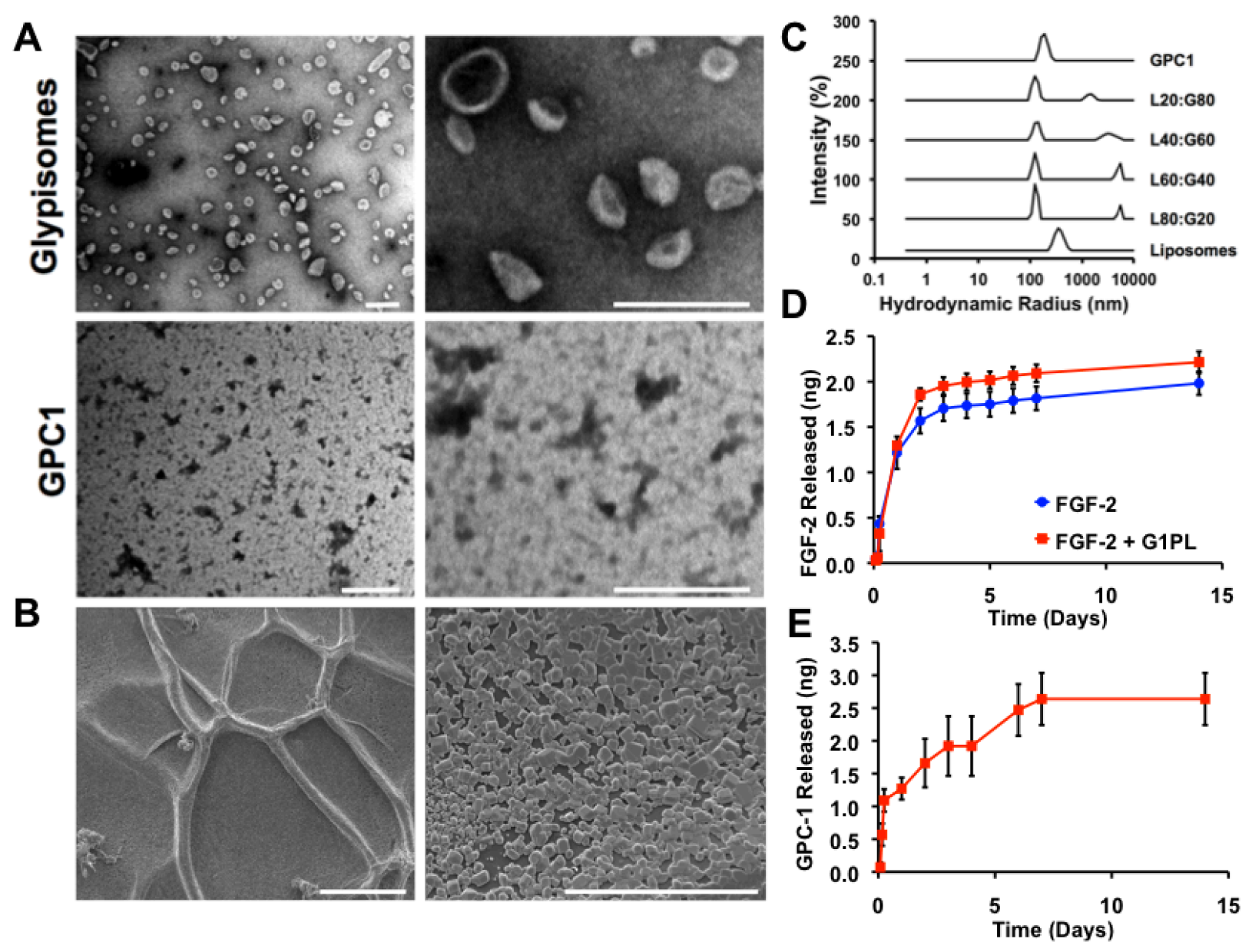

Page 23 of 29 
Figure 4

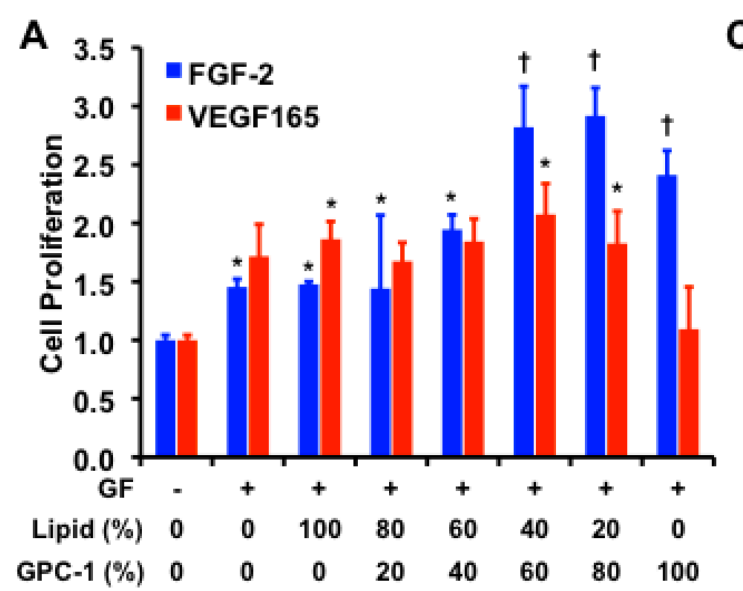

C Control

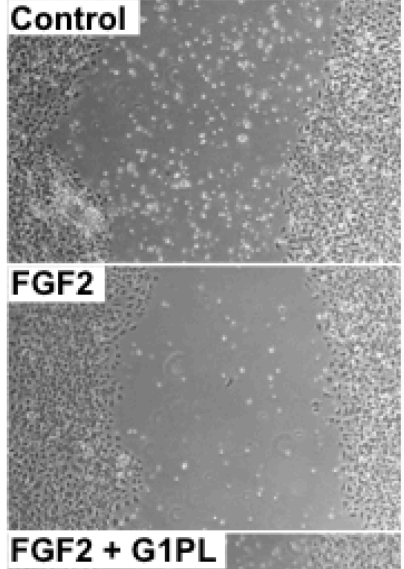

Control

B
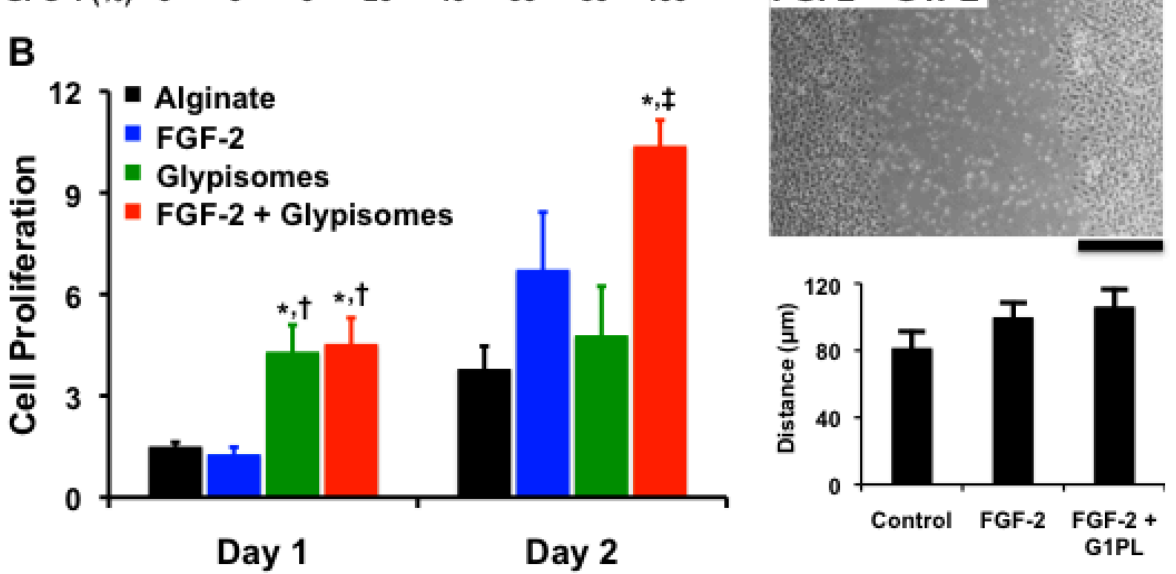

VEGF $_{165}+\mathrm{G1PL}$
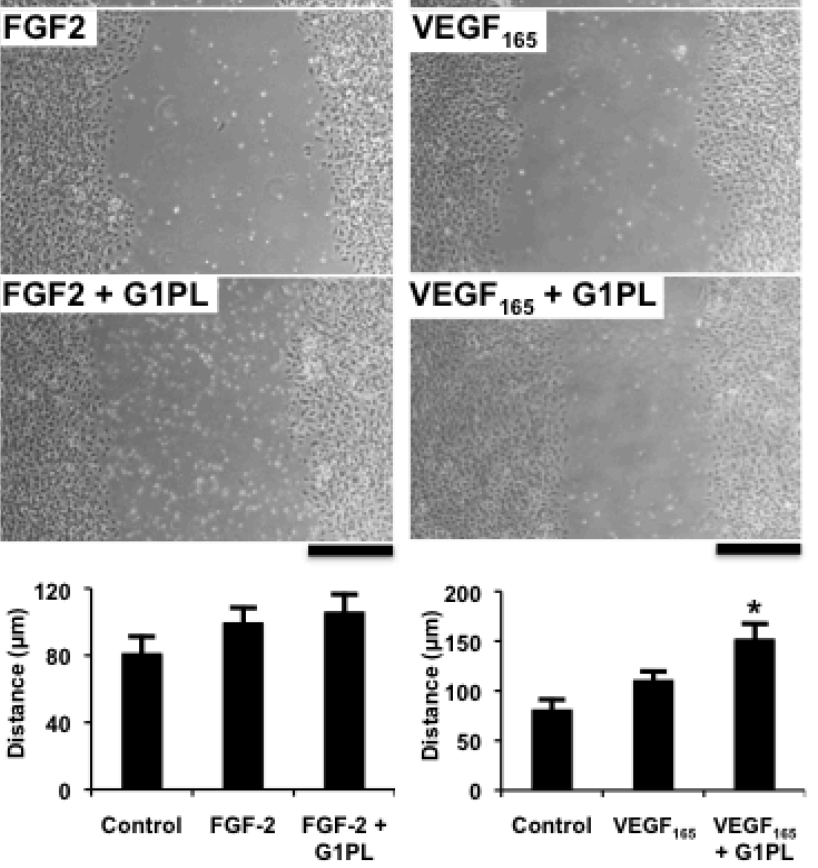
Figure 5

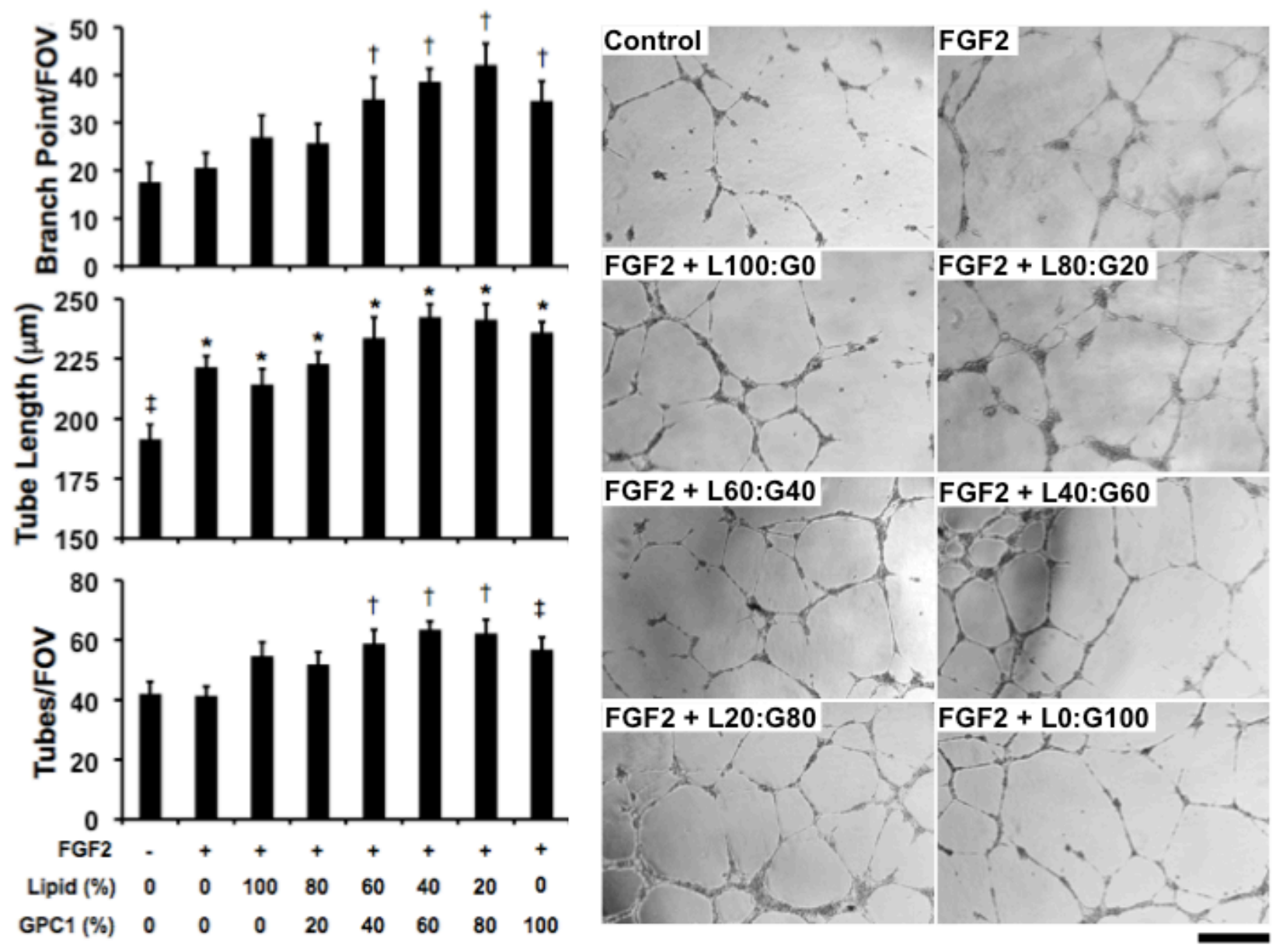


Figure 6
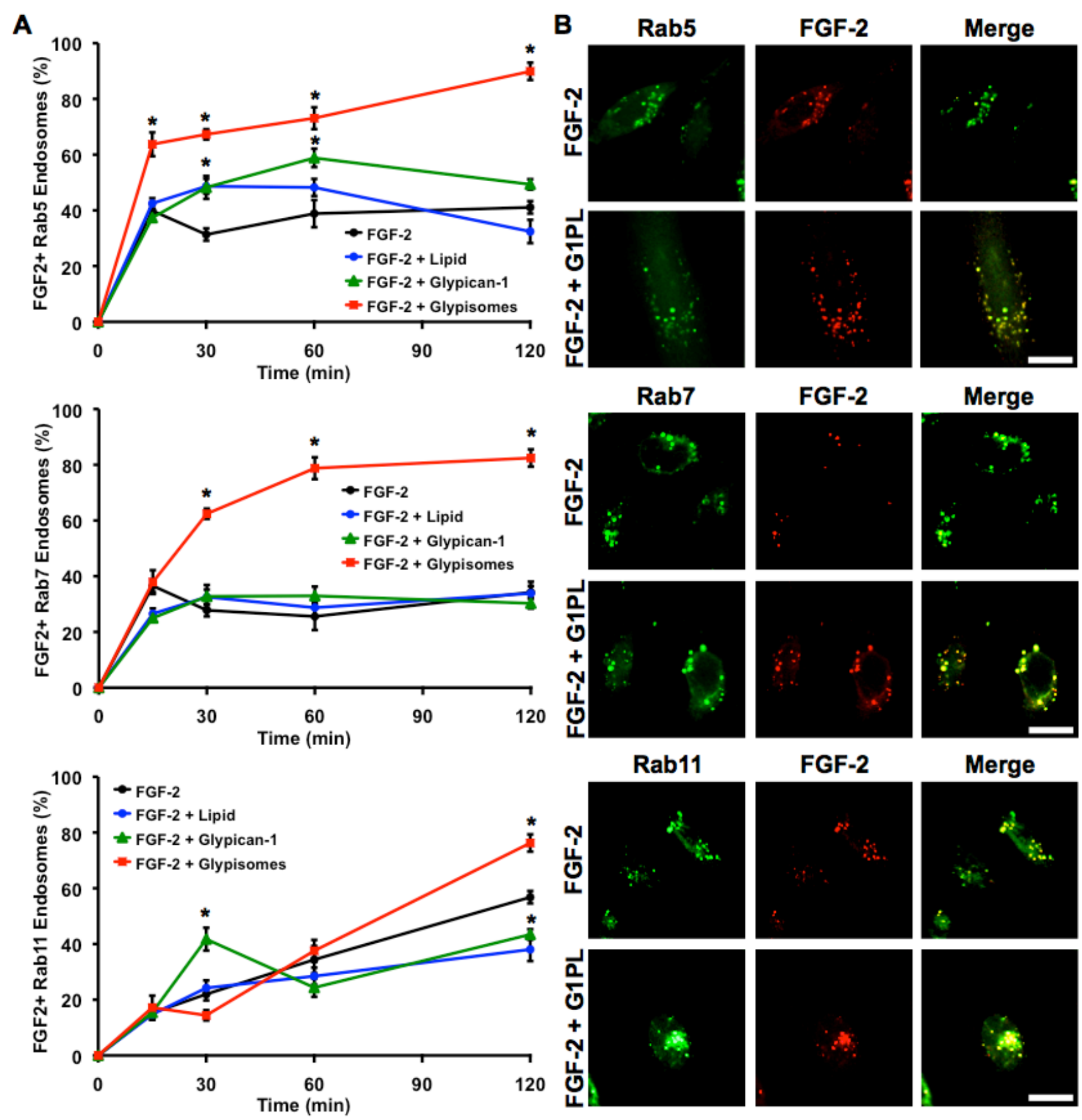

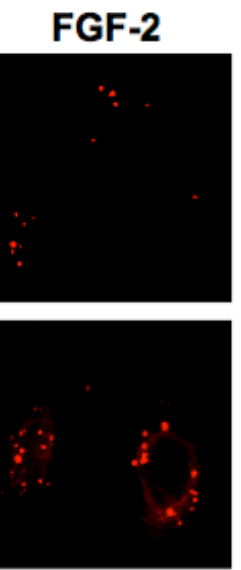

FGF-2

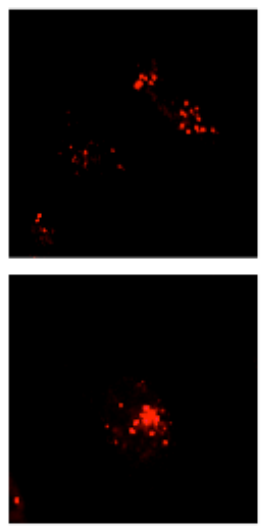

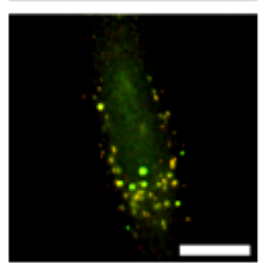

Merge
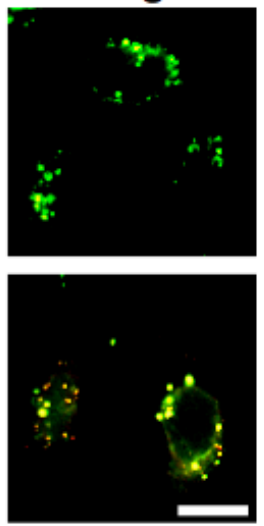

Merge
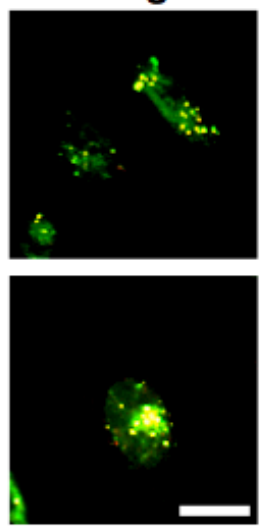
Figure 7
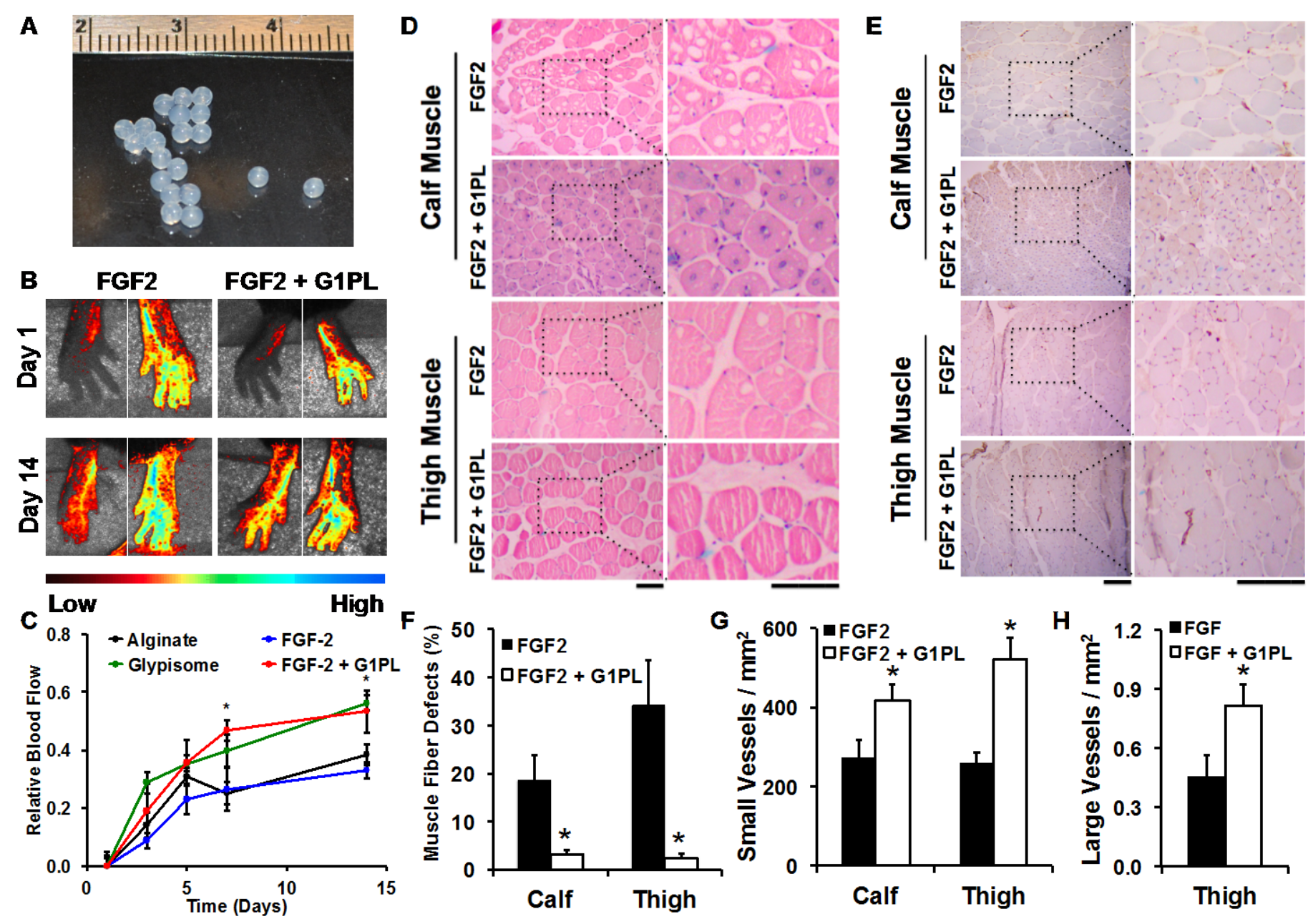
Figure 8
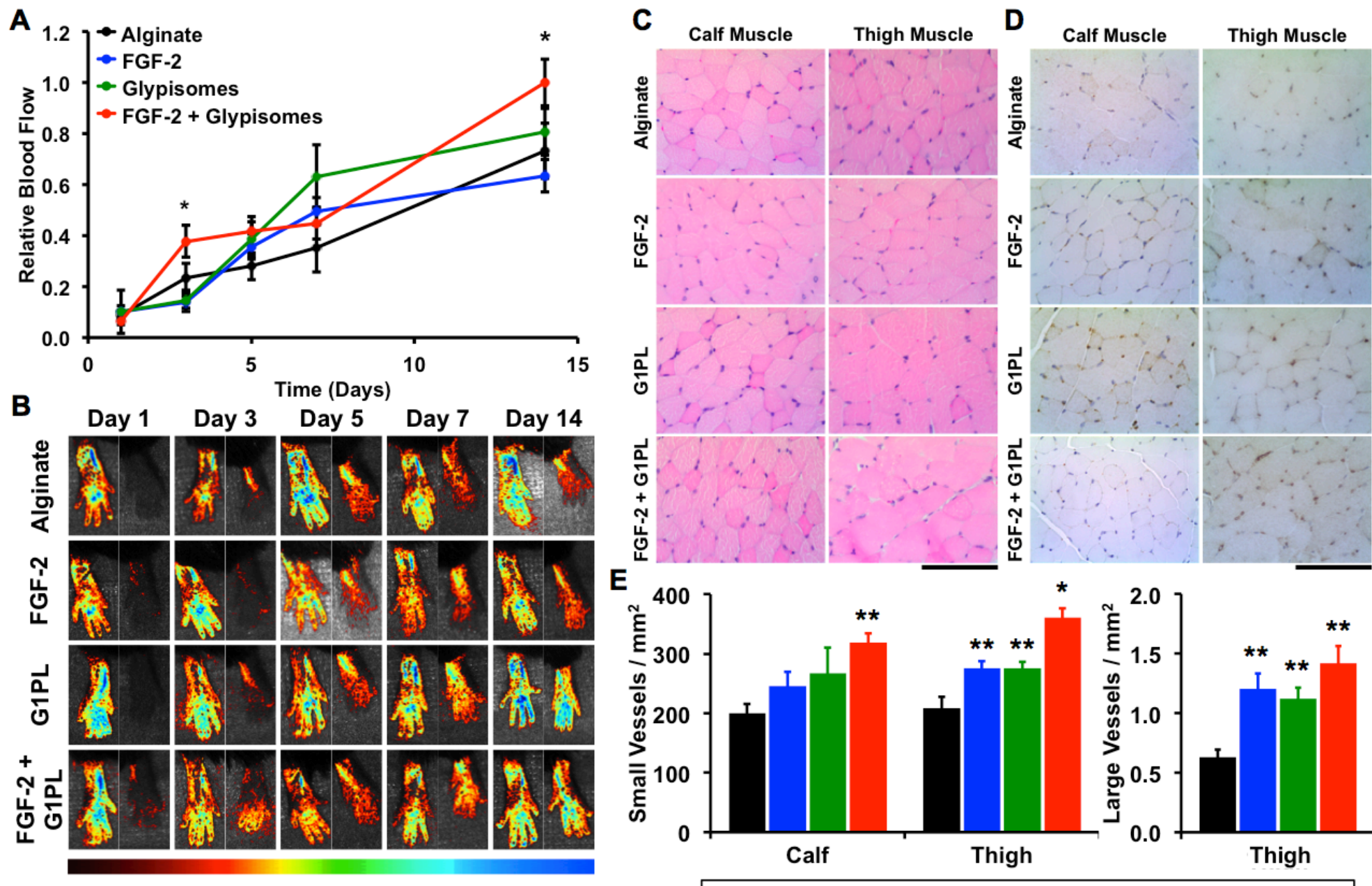

E
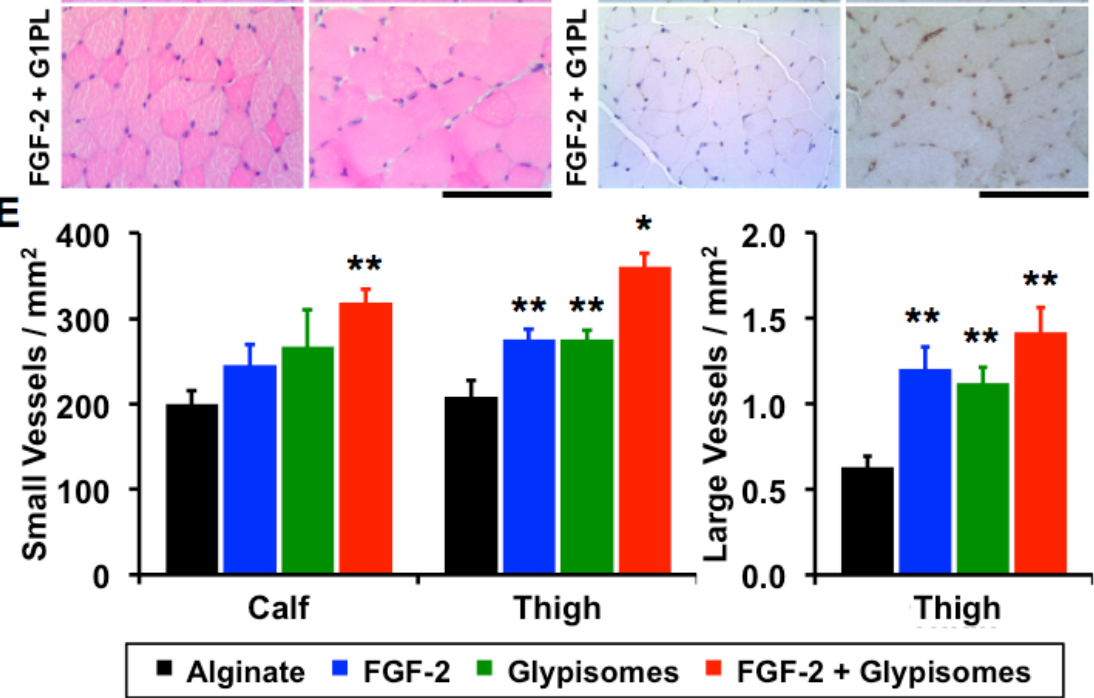


\section{Table 1}

Table 1. Patient Sample Data

\begin{tabular}{ccc}
\hline Diabetes Status & Age & Sex \\
\hline Normal & 59 & $\mathrm{M}$ \\
Normal & 49 & $\mathrm{~F}$ \\
Normal & 69 & $\mathrm{M}$ \\
Normal & 62 & $\mathrm{M}$ \\
Normal & 80 & $\mathrm{~F}$ \\
Normal & 39 & $\mathrm{~F}$ \\
Diabetic, type II & 73 & $\mathrm{M}$ \\
Diabetic, type II & 47 & $\mathrm{~F}$ \\
Diabetic, type II & 80 & $\mathrm{M}$ \\
Diabetic, type II & 81 & $\mathrm{M}$ \\
Diabetic, type II & 53 & $\mathrm{M}$ \\
Diabetic, type II & 61 & $\mathrm{M}$ \\
Diabetic, type II & 67 & $\mathrm{M}$ \\
\hline
\end{tabular}

